$(\mathrm{p}<0.10)$ with 90 -day hospital readmission. In all models, patients were stratified by age group ( $<65$ and $\geq 65$ years) to account for differences in Medicare eligibility. Results: Among all patients analyzed, 4,391 self-identified as AA and 63,625 self-identified as white. Among those $<65$ years of age, AAs, in comparison to whites, were less likely to have private insurance $(52.5 \%$ vs. $82.7 \%)$ and more likely to rely on Medicare or Medicaid $(47.5 \%$ vs. $17.3 \%$ ) (both $\mathrm{p}<0.001)$. For those age $\geq 65, \sim 90 \%$ of both AAs and whites relied on Medicare. Co-morbidities such as hypertension, diabetes and renal failure were more common among AAs than whites ( $p<0.001$, all comparisons, both age groups).

The Figure summarizes the unadjusted (UN) and adjusted (ADJ) RRRs of referral to an IRF, SNF and HH care (vs. home self-care) in AAs (vs. whites) by age group. Among patients $<65$ years of age, compared to whites, AAs had higher risk of discharge to an IRF (aRRR 2.56, 95\% Cl, 1.77-3.71) and a SNF (aRRR 3.37, $95 \% \mathrm{Cl}, 2.07-5.49$ ). Among those $\geq 65$ years of age, AA patients also had higher risk of discharge to an IRF (aRRR 1.96, 95\% Cl, 1.39-2.76) and a SNF (aRRR $3.66,95 \% \mathrm{Cl}, 2.29-5.84)$. Discharge to either IRF ( $<65$ years: aOR $4.06,95 \% \mathrm{Cl}$, $3.49-4.74 ; \geq 65$ years: aOR $4.32,95 \% \mathrm{Cl}, 3.67-5.09$ ) or SNF ( $<65$ years: aOR $2.05,95 \% \mathrm{Cl}, 1.70-2.46$; aOR $1.74,95 \% \mathrm{Cl}, 1.46-2.07$ ), instead of home with self-care, was significantly associated with higher odds of hospital readmission within 90 days.

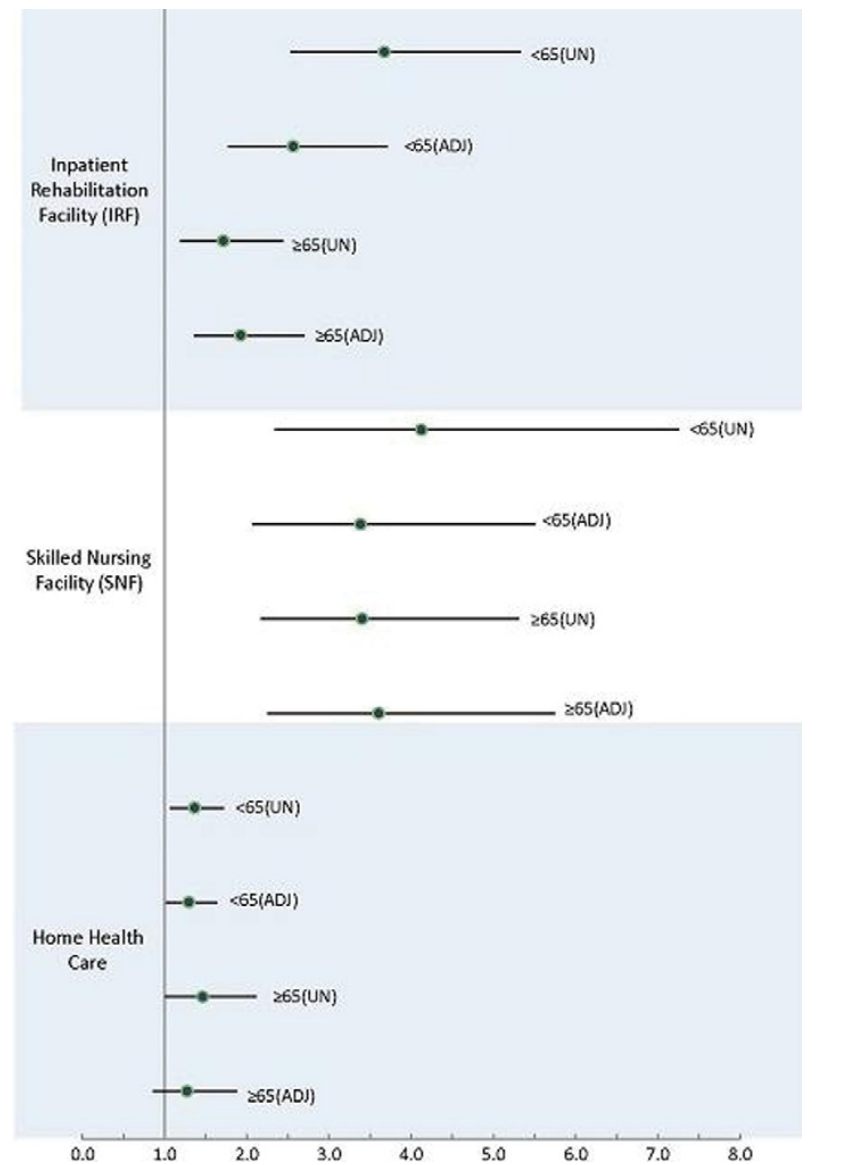

Conclusions: Compared to whites, AA patients who underwent hip replacement were more likely to be discharged to an IRF or SNF. Furthermore, discharge to either IRF or SNF was associated with higher risk of hospital readmission.

Disclosure of Interest: None declared

DOI: 10.1136/annrheumdis-2017-eular.2168

\section{THU0708 LONG-TERM OUTCOME AND RISK FOR TOTAL ARTHROPLASTY OF KNEE OSTEOARTHRITIS AFTER COMPREHENSIVE REHABILITATION}

F. Angst $^{1,1}$, T. Benz ${ }^{1}$, A. Aeschlimann ${ }^{1}$, S. Lehmann ${ }^{1}$, B. Winteler ${ }^{2}$, M.L. Verra ${ }^{2}$. 'Research, Rehabilitation Clinic "REHACLINIC", Bad Zurzach; ${ }^{2}$ Physiotherapy, Bern University Hospital, Bern, Switzerland

Background: Knee osteoarthritis affects mobility leading to substantial loss of perceived health and quality of life. Our previous study showed that aged persons with various comorbidities may profit from comprehensive rehabilitation in the short-term by corrected effect sizes up to 0.62 in pain, 0.51 in physical function, and 0.32 in psychosocial health (1).

Objectives: To follow-up those patients to 5 years by 1) quantifying observed effects in pain, function, and psychosocial health and 2) associating risk factors to total knee arthroplasty during the observation period.
Methods: Prospective cohort study with assessments at baseline (start of rehabilitation) and 1, 2, 3, 4, 5 years after. Comprehensive rehabilitation lasted 23 weeks for inpatients and 6 weeks for outpatients. Changes between baseline and the follow-ups were measured by the Western Ontario and McMaster Universities Osteoarthritis Index (WOMAC) and the Short Form 36 Health Survey (SF-36). They were expressed as effect sizes (ES) according to Kazis (2). Multivariate logistic regression included various sociodemographic and disease-modifying confounders and provided adjusted odds rations $(\mathrm{OR})$ for the risk of getting total knee arthroplasty.

Results: At baseline, $\mathrm{n}=205$ knee osteoarthritis patients were included: $77.1 \%$ women, mean age 65.7 years $(\mathrm{sd}=10.3 \mathrm{y}), 81.5 \%$ having 3 or more comorbidities. Up to the 5 year follow-up, $n=83(40.5 \%)$ remained with complete data, 48 (23.4\%) received arthroplasty. At 5 years, ES were 0.13 to 0.79 for pain, -0.12 (worsening) to 0.42 (improvement) for function, and -0.25 to 0.21 for psychosocial health. At the last follow-up before surgery, WOMAC pain had worsened by $E S=-$ $0.42(\mathrm{p}=0.001)$ and WOMAC function by $E S=-0.54(\mathrm{p}=0.002)$ in the total knee arthroplasty group. Getting total knee arthroplasty was statistically significantly associated with female sex $(O R=3.30)$, educated at university $(O R=3.54)$, minus 1 comorbidity less $(\mathrm{OR}=1.41$ ), and 10 (of 100 possible) points worsening on the WOMAC factor ascending/descending (OR=1.60).

Conclusions: Moderate to small improvements on pain, function, and psychosocial health were observed up to 5 years after comprehensive rehabilitation of knee osteoarthritis. Nevertheless, almost one quarter of the participants were referred to total knee arthroplasty suffering from significant deterioration in pain and function. The WOMAC seems to be sensitive to predict the need for arthroplasty. Highly educated women with low number of comorbidities and high disability to manage stairs were more likely to receive total knee arthroplasty (3).

References:

[1] Angst F, Verra ML, Lehmann S, Benz T, Aeschlimann A. Effects of inpatient rehabilitation in hip and knee osteoarthritis. A naturalistic prospective cohort study with intra-individual control of effects. Arch Phys Med Rehabil 2013;94;2139-45.

[2] Kazis ES, Anderson JJ, Meenan RF. Effect sizes for interpreting changes in health status. Med Care 1989;27(3 Suppl):S178-89.

[3] Verra ML, Benz T, Lehmann S, Aeschlimann A, Winteler B, Angst F. Long-term outcome of knee osteoarthritis after comprehensive rehabilitation - 5 year follow up and risk for total knee arthroplasty. Journal 2017; in preparation.

Disclosure of Interest: None declared

DOI: 10.1136/annrheumdis-2017-eular.1822

\section{THU0709 DOES THE USE OF ANALGESIC CURRENT THERAPIES INCREASE THE EFFECTIVENESS OF NECK STABILIZATION EXERCISES FOR IMPROVING PAIN, DISABILITY, MOOD, AND QUALITY OF LIFE IN CHRONIC NECK PAIN? A RANDOMIZED, CONTROLLED, SINGLE-BLIND STUDY (A PILOT STUDY)}

H. Yesil $^{1}$, S. Hepguler ${ }^{2}$, U. Dundar ${ }^{1}$, S. Taravati ${ }^{2}$, B. Isleten ${ }^{3} .{ }^{1}$ Physical Medicine and Rehabilitation, Afyon Kocatepe University, Faculty of Medicine, Afyonkarahisar; ${ }^{2}$ Physical Medicine and Rehabilitation, Ege University, Faculty of Medicine, Izmir; ${ }^{3}$ Physical Medicine and Rehabilitation, Yalova Aktif Hastanesi, Yalova, Turkey

Background: Analgesic therapies; such as interferential current (IFC) and transcutaneous electrical nerve stimulation (TENS) have been applied solo or combined with exercise for management of neck pain (NP), however, the efficacy of these combinations are unclear.

Objectives: To determine if TENS or IFC increase the effectiveness of neck stabilization exercises on pain, disability, mood, and quality of life for chronic NP. Methods: 60 patients with chronic NP were included in the study. Patients were randomly assigned into 3 groups; Group I: neck stabilization exercise, Group II: TENS+ neck stabilization exercise and Group III: IFC+ neck stabilization exercise. Patients' pain levels (visual analogue scale (VAS)), quality of life (short form36 ), mood (Beck depression inventory (BDI)), levels of disability (Neck Pain and Disability Index) and the need for analgesics were evaluated prior to treatment, at 6 th and 12th week follow-up. All participants had group exercise accompanied by a physiotherapist for 3 weeks and an additional 3 weeks of home exercise program.

Results: All three groups had statistically significant improvement regarding their VAS, neck disability index and most sub-scores of short form-36 $(p<0,05)$. At 12th- week follow-up, no difference was found between groups regarding pain, disability, and quality of life $(p>0,05)$. On the other hand, analyses indicated significantly lower scores of neck disability index $(p=0.004)$ and less need for paracetamol $(p=0.036)$ in TENS group 6th-week follow-up when compared to exercise and IFC groups.

Conclusions: All treatment modalities improved pain, disability, mood and quality of life in patients with chronic NP. Besides, results suggest TENS enhanced the efficacy of exercise therapy on pain, disability and need for analgesics in acute phase but not in long term, since there was no difference at 12 th week follow-up. To conclude, we think exercise protocols can be of choice since they are inexpensive, easy and effective in the management of chronic NP.

References:

[1] Acedo AA, Luduvice Antunes AC, Barros dos Santos A, Barbosa de Olveira C, Tavares dos Santos C, Colonezi GL, Fontana FA, Fukuda TY. Upper trapezius 